\title{
Unmanned Aerial Vehicle Antenna Measurement Using Anechoic Chamber
}

\author{
Elena M. Dobychina, Mikhail V. Snastin, Evgeniy N. Efimov, Timofey Y. Shevgunov \\ Moscow Aviation Institute (National Research University),Volokolamskoe Highway, 4, \\ Moscow, 125993, Russia
}

\begin{abstract}
Anechoic chambers (AECs) are increasingly used by research centers to obtain the characteristics of antenna systems necessary to compare them with the results of electrodynamics modeling. A review of the current publications on this topic has shown an increased interest in the general problems of AEC design, the choice of the shape and material of radar absorbing coatings that minimize reflections inside the chamber, as well as in the methods for measuring various antenna characteristics.
\end{abstract}

Keywords - anechoic chamber, antenna gain, antenna radiation pattern, drone, near field scanner, unmanned aerial vehicle, antenna array

\section{Introduction}

Devices that work independently or controlled by an operator at a distance take an increasing place in human life and activities. Among their diversity unmanned aerial vehicles (UAVs) are the most in demand in all areas of activity. They are especially necessary for monitoring and ensuring the safety of the construction and operations at oil and gas production platforms and at other places that are hardly accessible or even hazardous. On the UAV board there must be a specialized radio electronic system (RES).

DOI: $10.18421 /$ TEM94-21

https://doi.org/10.18421/TEM94-21

Corresponding author: Timofey $Y$. Shevgunov, Moscow Aviation Institute (National Research University), Moscow, Russia.

Email: shevgunov.timofey@mail.ru

Received: 07 September 2020.

Revised: 02 November 2020.

Accepted: 07 November 2020.

Published: 27 November 2020.

(c) BY-NC-ND (c) 2020 Elena M. Dobychina et al; published by UIKTEN. This work is licensed under the Creative Commons Attribution-NonCommercial-NoDerivs 4.0 License.

The article is published with Open Access at www.temjournal.com
Multielement phased antenna arrays (PAA) with spatial-temporal signal processing algorithms applied to the tasks of UAV control, radio navigation, surveillance and telecommunications are an integral part of the RES. Radar system operates in the presence of various noises and disturbances and performs digital signal processing of random processes that are often non-stationary [1] processes or other processes that require special approaches and modeling techniques [2]. Antenna array (AA) is built based on digital transceiver modules [3] that analyze the scattering and reflections of the observed objects. The characteristics of the antenna array must match that of technical specification with various measurement procedures and calibration techniques used to establish, control and verify that state [4]. Measurements of antenna characteristics are traditionally carried out at open antenna test facilities dedicated for such tests; these facilities try to provide operating conditions for the measurements in the far field (FF) even for large antenna arrays. The main difficulty in carrying out such experiments is the multi-beam propagation nature of radio waves; due to the influence of the underlying surface of the earth. An interference occurs for radiating antenna and antenna under test are linked not only by the direct beam connecting phase centers but also by the beam that reflects from the surface of the earth. The use of an anechoic chamber (AEC) can significantly reduce or completely eliminate the need of testing at the specialized antenna test facilities that in turn leads to a significant increase in the accuracy of the measurements saving money and time that otherwise are spent on developing and maintaining specialized antenna test facilities in working condition [5].

High requirements are imposed on the AEC characteristics to ensure small values of the measurement error [6]. A chamber is typically characterized by its physical size, shield performance and reflectivity level. The chamber itself is a closed electromagnetic shield, the inner surfaces of which have a regular or special shape and are fully or partially covered with a radar absorbing material (RAM).

The reflectivity level is primarily determined by the properties of the RAM used in the AEC. 
Absorbent materials generally have a known reflectivity in case of a normal wave incidence of propagating electromagnetic waves. However, in most indoor applications it is important to know their bistatic reflectivity for oblique angles of arrival of the waves [7]. Many scientific articles discuss the absorbing and scattering properties of various RAMs employing theoretical approaches and approximation procedures to model complex processes occurring in such materials. These are polynomial equations for estimation of the reflected energy [8], scaling models [9], electrodynamic models of processes occurring in multilayer structures [10] and low frequency models for pyramidal panels [11]. Also, noteworthy are recent experimental studies of radio absorbers based on organic materials [12] and metamaterials [13].

The use of AEC allows measurements of the antenna radiation pattern (ARP) characteristics in the FF for the case of appropriate ratios of the dimensions of the chamber to the dimension of the antenna and to the operating frequency. For antenna arrays of large UAVs, the size of the far filed range is typically tens or even hundreds of meters and in that case the use of AEC becomes the only option for it allows carry out measurements in the near filed region using planar [14], cylindrical or spherical scanning.

All experiments presented in the paper were carried out in the AEC. The goals of this study are the demonstration of the main capabilities of AEC created and deployed as an innovative project [15] at the national research university and the measurement of the main antenna characteristics by the near-field techniques using the as an example the antennas for UAVs and compare the results with the reference data obtained at open specialized antenna test facilities and corresponding manufacturer certificates.

\section{Theoretical Background}

Near filed measurement method is conceptually based on the uniqueness of the solution of the exterior electrodynamics problem [16]. The following statement reflects the essence of the theorem postulating the uniqueness of the solution of the exterior electrodynamics problem applied to the antennas. Complex FF scattering pattern is uniquely determined by the complex near field (NF) distribution on the scanning surface that covers antenna under test (AUT). In practice, the NF scanning surface is chosen to be flat, spherical, cylindrical and even conical.

The next theoretical principle used to solve the problem as it was previously formulated is the Huygens principle [17] that allows to calculate the FF scattering pattern as superposition of the secondary fields that have determined tangential parts for the given surface.

For the flat shape of the scanning surface $S$, the FF pattern described by the following analytical expression (the time factor $e^{j \omega t}$ is omitted in all expressions to obtain more compact forms):

$$
\dot{E}(\theta, \varphi)=j \frac{(1+\cos \theta) e^{-j k r}}{2 \lambda r} \iint_{S} \dot{E}(x, y) e^{j k \sin \theta(x \cos \varphi+y \sin \varphi)} d x d y
$$

where $d x d y=d S$ denotes surface element with coordinates $x$ and $y$ on the scanning projection $X O Y$; $\dot{E}(x, y)$ is the tangential component of the electrical field at the probe point; $\dot{E}(\theta, \varphi)$ is a complex FF pattern of the antenna on the sphere of radius $r$ in the directions of $\theta$ and $\varphi ; r$ is the distance from the origin on the selected plane to a point where the field $\dot{E}(\theta, \varphi)$ is defined, $\theta$ and $\varphi$ are angles in a spherical coordinate system.

Actual problem of determining the FF pattern $\dot{E}(\theta, \varphi)$ is narrowed down to a problem of $\mathrm{NF}$ determination $\dot{E}(x, y)$. There is an important aspect that highlights the advantages of the FF reconstruction based on the NF measurements. Expression (1) shows that the reconstructed FF pattern $\dot{E}(\theta, \varphi)$ is in fact an integral characteristic of a set of NF measured data $\dot{E}(x, y)$. That means that even if some of the NF measurements introduce substantial individual measurement errors, the overall result of the reconstructed FF will not be strongly influenced. That aspect makes NF measurement approach potentially much more accurate than the other ones based on direct FF measurements at an outdoor antenna test ranges where instrumental measurement errors are often hardly avoidable.

\section{Methodology}

The experiments presented in the following paper were carried out with the AMCC based on AEC [18] with operating frequency range from 0.8 to $40 \mathrm{GHz}$.

AEC meets strict requirements for shielding performance and reflectivity level within quiet zone, which are given in Table 1. 
Table 1. AEC specifications

Operating frequency range Wall shielding, door shielding

Normal wave incidence reflection

Electromagnetic shielding

The integrated software for automated measurements implements the following key features: control and data collection from all measurement devices, data processing, calculation of AUT parameters, storage organization and visualization of measured and processed data. The internal control system with the detailed report containing status information about all physical and integrated software components is also available.

The laboratory complex provides automated measurements of the main electrical parameters of antennas, antenna systems and microwave devices in a wide frequency range, in the near field and FF ranges, including:

- complex (amplitude and phase) ARP;

- complex (amplitude and phase) distributions over the antenna aperture;

- antenna gain factor;

- antenna axial ratio;

- antenna phase center location;

- voltage standing wave ratio (VSWR) of antennas and microwave devices.

Figure 1. contains pictures of the AEC interior with radar absorbing material and measurement equipment. The control room and workplaces are shown in Figure 2.
$800 \mathrm{MHz}-40 \mathrm{GHz}$ at least $90 \mathrm{~dB}$ from $100 \mathrm{kHz}$ to $40 \mathrm{GHz}$ at least $-30 \mathrm{~dB}$ from $1 \mathrm{GHz}$ to $40 \mathrm{GHz}$; at least $-20 \mathrm{~dB}$ from $0.8 \mathrm{GHz}$ to $1 \mathrm{GHz}$ steel panels that welded together

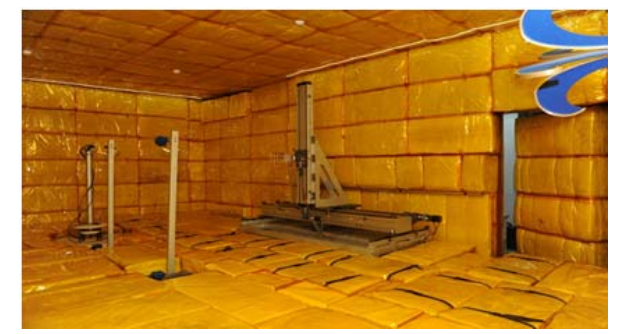

Figure 1. AEC with measurement equipment

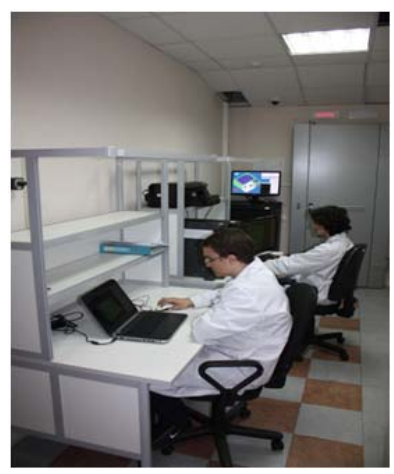

Figure 2. Control room interior

Antenna measurements are carried out with a vector network analyzer (VNA). To determine the amplitude and phase distribution of the electromagnetic field in the aperture of the AUT, a NF planar scanner [15] is used with specification given in Table 2.

Table 2. Scanner specifications

\begin{tabular}{lc}
\hline Size of the working area & $1.5 \mathrm{~m} \mathrm{x} 1.5 \mathrm{~m} \times 1.0 \mathrm{~m}$ \\
\hline Number of CNC axes & $4(\mathrm{X}, \mathrm{Y}, \mathrm{Z}, \mathrm{P})$ \\
\hline Accuracy of the probe positioning, linear coordinates & $\pm 0.1 \mathrm{~mm}$ \\
\hline Accuracy of the probe positioning, angular coordinates & \pm 0.15 degrees \\
\hline
\end{tabular}

Rotary antenna positioner (RAP) (Table 3.) is used to position antennas and antenna arrays during spatial and polarization ARP measurements in the FF region (Figure 3.).

Table 3. RAP specifications

\begin{tabular}{lc}
\hline Max weight load (dynamic) & $80 \mathrm{~kg} / \mathrm{m}^{2}$ \\
\hline Max weight load (static) & $200 \mathrm{~kg} / \mathrm{m}^{2}$ \\
\hline Support device for small-scale antennas, load & $7.5 \mathrm{~kg} / \mathrm{m}^{2}$ \\
\hline Number of CNC axes & $3(\mathrm{AZ}, \mathrm{EV}, \mathrm{P})$. \\
\hline Positioning accuracy, angular coordinates & $\pm 0.15^{\circ}$ \\
Positioning accuracy, linear coordinate Z & $\pm 0.1 \mathrm{~mm}$. \\
\hline & AZ axis: $\pm 180^{\circ} ;$ \\
Rotating angle & Phi axis: $\pm 180^{\circ} ;$ \\
& EL axis: $\pm 30^{\circ}$ \\
\hline
\end{tabular}


AMCC is equipped with fire extinguishing, video surveillance and access control systems. The AES equipment supports the required safety from electric overload and is sustainable to the external shock in power supply. The AMCC equipment is located in a separate shielded room (Figure 4.).

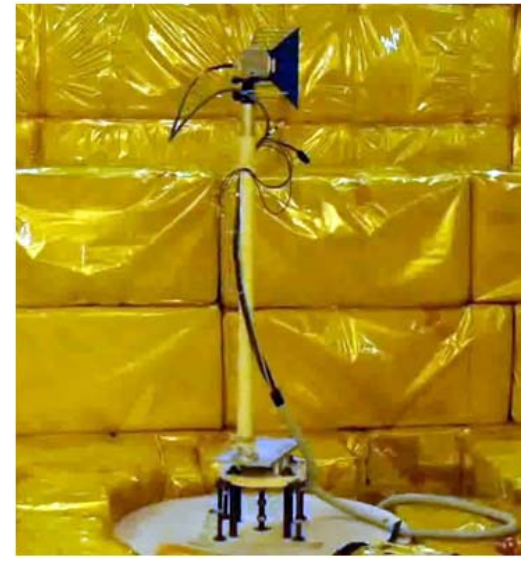

Figure 3. Antenna under test mounted on RAP

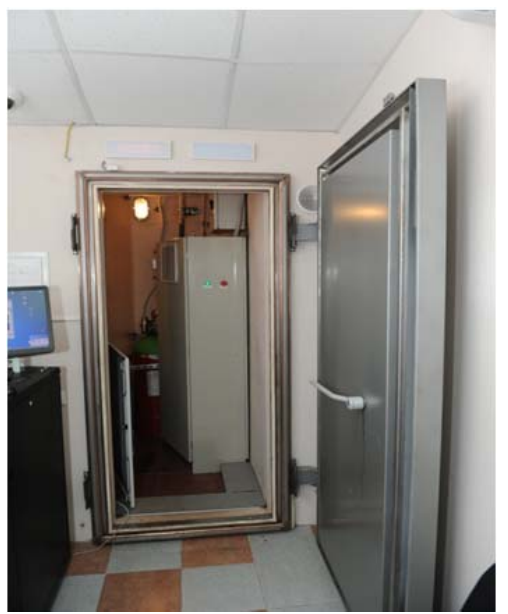

Figure 4. Equipment compartment

Antenna measurement techniques for conducting antenna measurements have been developed, taking into account the latest technical solutions used in the AMCC. These solutions allow fully automated measurement of the characteristics of antenna systems parameters in range from $800 \mathrm{MHz}$ up to 40 $\mathrm{GHz}$ in a single measurement cycle with no need of additional manual equipment reconfiguration.

The results are uploaded to a PC workstation and can be used both to prepare in measurements reports or and to in perform additional calculations and further modeling.

\section{Results}

The AEC is capable of performing a various range of measurements, for example, polarization characteristics of objects; a reflectivity of complex shape objects may be measured as function of various antenna-object positions and orientations.
This paper outlines the capabilities of AMCC for measurements of high-RF path parameters and directivity and frequency characteristics of aperture antennas and antenna arrays.

Experimental prototype of antenna array (AA) for UAV was modeled, developed and manufactured. The presented AMCC made it possible to add one more stage to the device development cycle, namely the measurement stage, which is preceded by the electrodynamic modeling, development of design documentation, schematics design and production stage. The experimental study of the AA characteristics was carried out by near field measurement process with the following reconstruction of the far field using (1). Equipment set used for measurements is shown in Figure 5. AUT is mounted on a tripod in front of the NF scanner at a distance of 2 to 3 wavelengths, between AUT and the probe located on scanner (Figure 6.).

$\mathrm{S}$-parameters are measured in a wide frequency range using VNA. The whole process is automated and controlled by an operator using a PC workstation with specialized software.

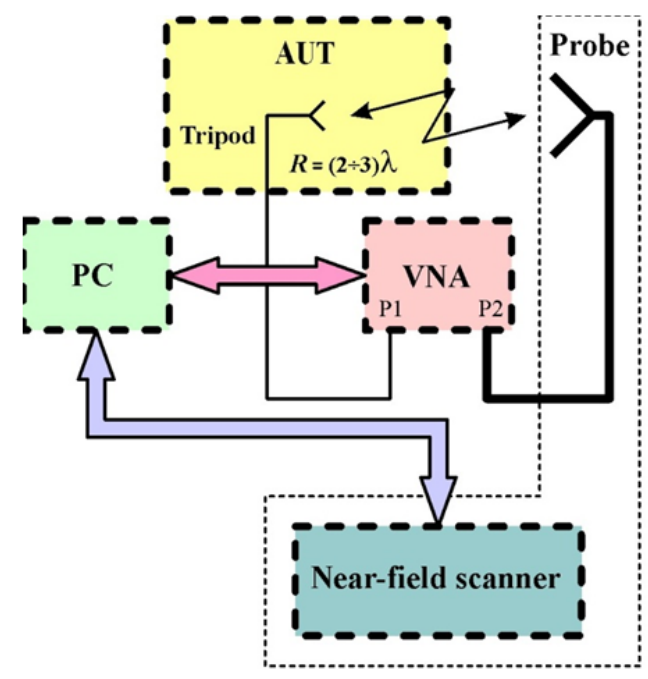

Figure 5. Measuring setup concept scheme

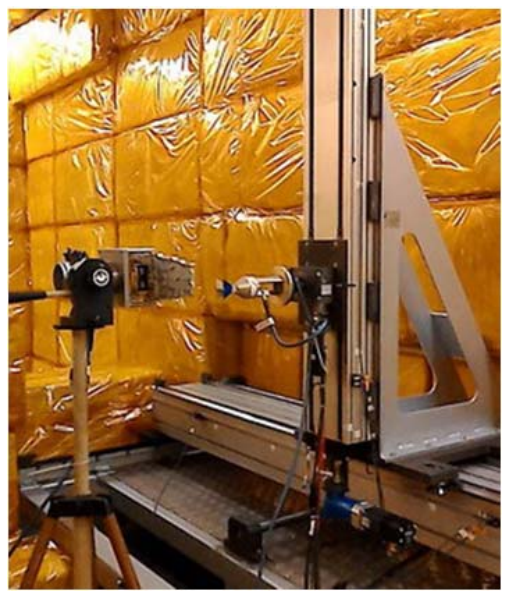

Figure 6. AA located in front of scanner 
The measurement result is the NF amplitude distribution in the scanning plane of AUT (Figure 7., Figure 8.). Phase distribution may be used as well if necessary.

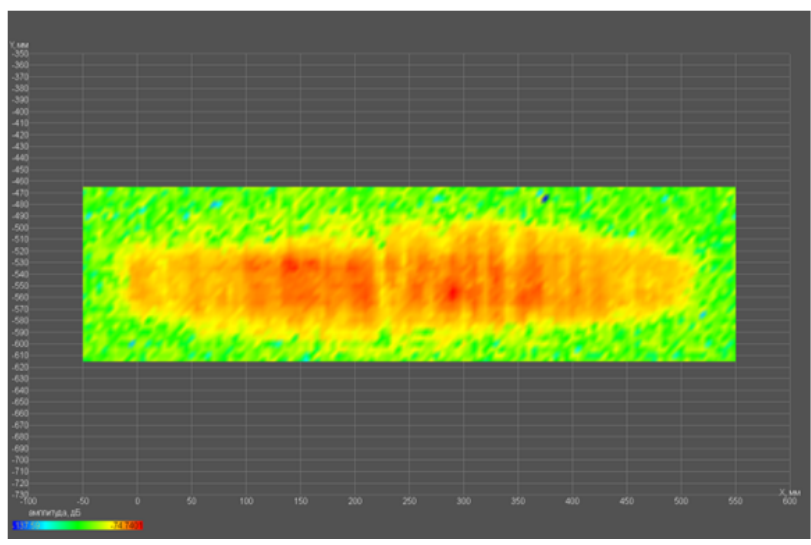

Figure 7. NF amplitude distribution
Fourier transform used for performing FF reconstruction.

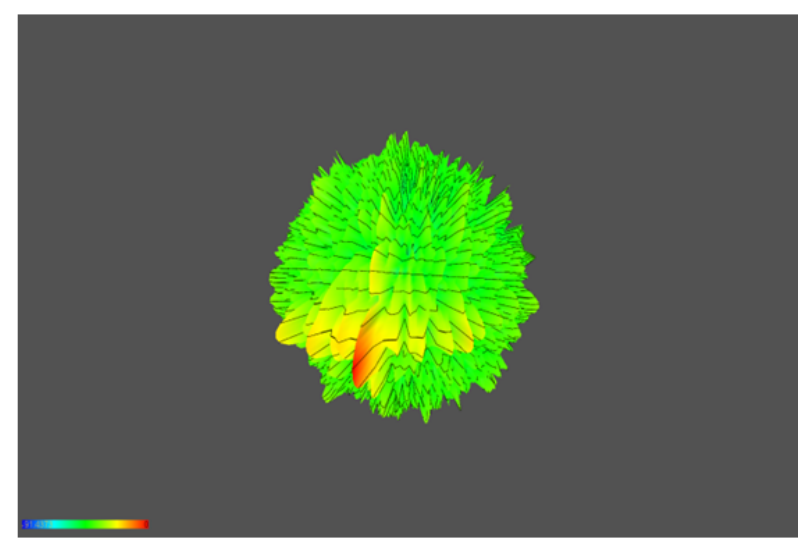

Figure 8. 3D image of NF amplitude distribution

Reconstructed FF ARP is shown in Figure 9. and Figure 10.

dB

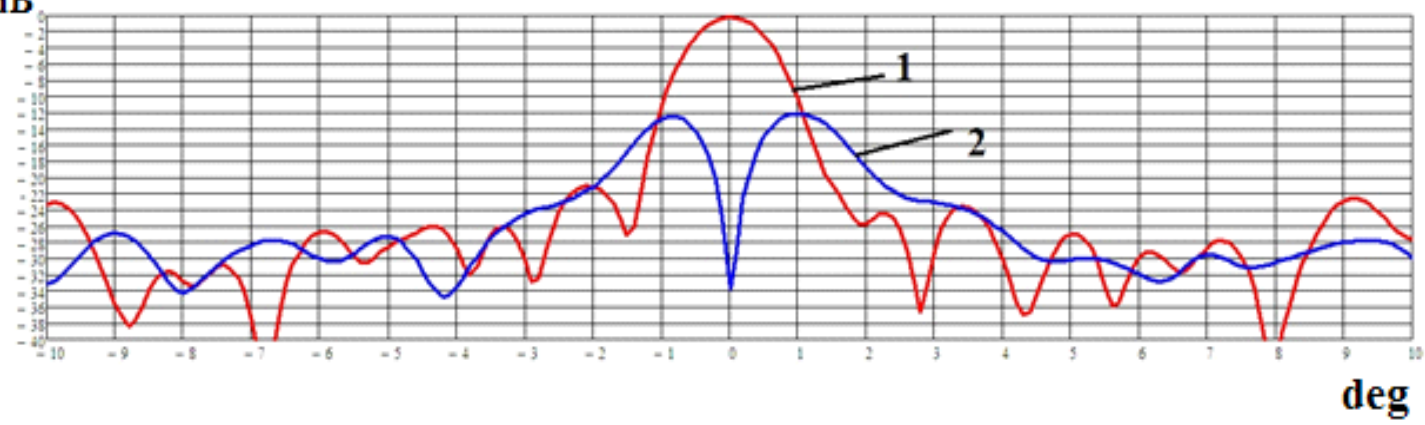

Figure 9. ARP in azimuthal plane: 1- AA sum channel; 2- AA difference channel

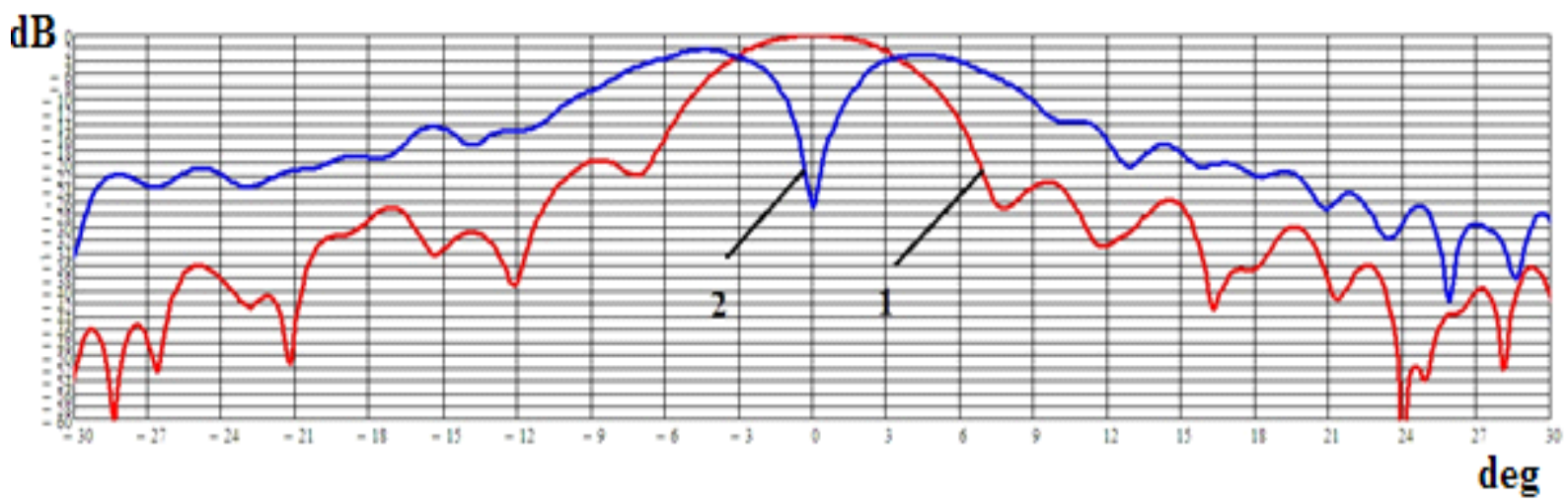

Figure 10. ARP in elevation plane: 1- AA sum channel; 2- AA difference channel

The resolution of the conversion result is $0.1^{\circ}$.
The measured VSWR for the AA transmitting input is shown in Figure 11.

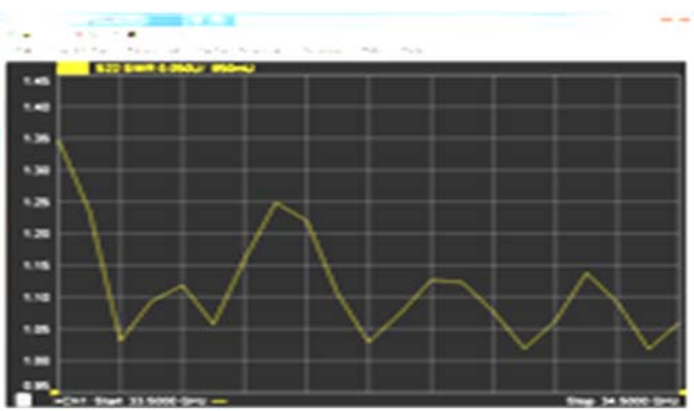

Figure 11. VSWR within the frequency range 


\section{Discussion}

To verify the results of NF measurements, a comparison was carried out with similar FF measurements obtained during the tests of the same antenna array at the outdoor antenna test facility. Table 4. contains the comparison of main parameters.

Table 4. Trial results

\begin{tabular}{lcccc}
\hline \multirow{2}{*}{ Parameter name } & \multicolumn{2}{c}{ Outdoor test facility } & \multicolumn{2}{c}{ AEC } \\
\cline { 2 - 5 } & H-plane & E-plane & H-plane & E-plane \\
\hline Gain, dB & 30.4 & 30.4 & 31.1 & 31.1 \\
\hline Beam width (sum channel), $^{\circ}$ & 6.2 & 1.16 & 6.4 & 1.17 \\
\hline Sidelobe level, dB $_{\text {Null level (relative to sum channel), dB }}$ & -23.2 & -21.0 & -22.9 & -20.6 \\
\hline AA input VSWR & -28.0 & -35.0 & -27.1 & -33.9 \\
\hline
\end{tabular}

The following parameters calculated via NF to FF transformation correspond to similar characteristics measured directly on far field: beam width of the sum ARP in the horizontal and vertical planes, null level of difference pattern, and AA gain. That correspondence proves the validity of the AEC measurements.

A test setup was used in automated MCC to measure antenna gain with Purcell's mirror method (Figure 12.). The idea of this method is to compare the reflection coefficients of the AUT for two cases: free space radiation and radiation in the direction of reflective flat shield or other reflector positioned at the distance $R$. The mismatch is caused by reflector affects to AUT input impedance. The initial input impedance is changed by the additive impedance induced by the reflector. That addition is equivalent to the impedance induced by the similar antenna as AUT located at a distance of $2 R$ and having a reverse phase.

The measured gain with investigated method does not depend on the influence of the ground surface since given distance $R$ is small and the reflected wave can be neglected.

However, it depends on the degree of AUT matching and on internal wall reflections within AEC. The following expression is used for the gain $(G)$ corresponding to reflection coefficient of the mismatched AUT:

$$
G=\frac{\left|\Gamma_{R}-\Gamma_{0}\right|}{1-\left|\Gamma_{0}\right|^{2}} 4 k R,
$$

where $\Gamma_{R}$ - reflection coefficient measured with the reflector in front of AUT, $\Gamma_{O}$ - reflection coefficient measured in free space.

In some cases, the UAV radio system includes aperture antennas, such as horn, spiral, or open ends of the waveguide. Therefore, to measure the gain using the described method a wide-band horn antenna was used in the experiment. It was placed on a planar scanner (Figure 13.) and the distance to the reflector was changed by high-precision movement along the Z-axis of the scanner.

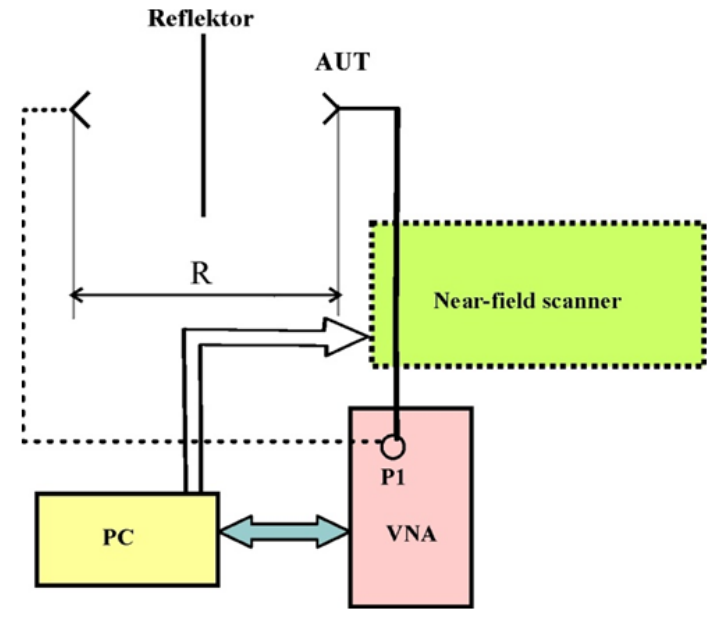

Figure 12. Gain measure concept

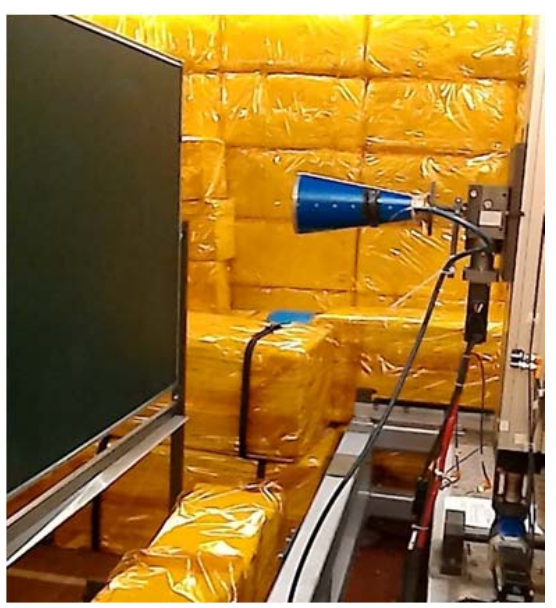

Figure 13. AUT in front of the reflector

The measurement of the complex reflection coefficient in a wide frequency range was carried out using a vector network analyzer. The whole process was carried out in a full automatic manner and was supervised by an operator using a PC workstation. The frequency dependence of the AUT gain was obtained and shown in Figure 14. The solid line 1 stands for the measurement results and the dashed line 2 stands for the data from the antenna calibration certificate. 


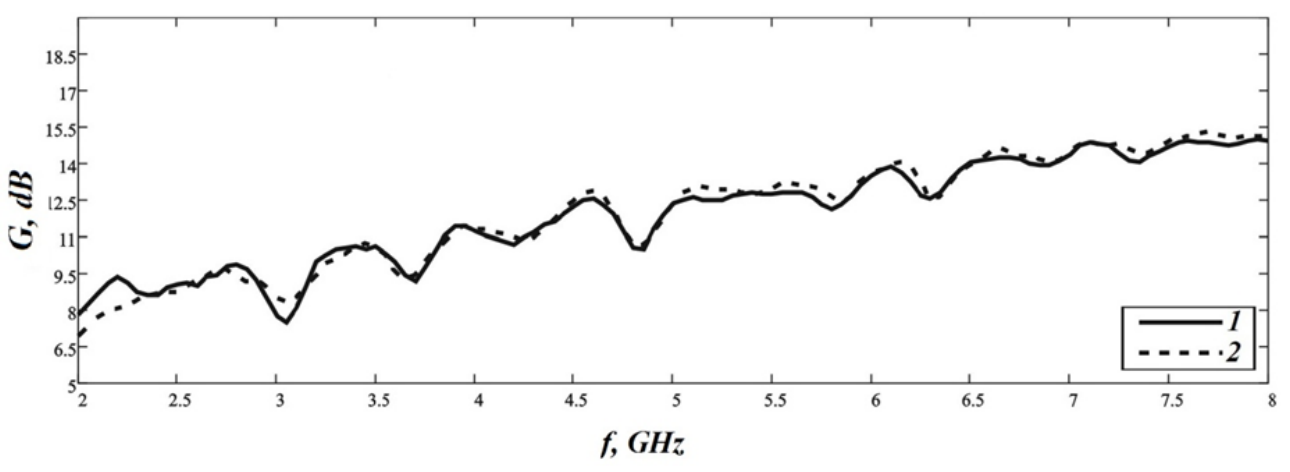

Figure 14. Gain in the operating frequency range

The experimental data are in good agreement with the AUT calibration certificate; the maximum gain deviation is $0.67 \mathrm{~dB}$ at low frequencies over the entire measured band.

During experiments, the method of measuring the parameters of antennas that does not require a FF range was implemented, and the high accuracy of measurements was obtained.

\section{Conclusions}

In this paper, it was shown that experimental studies of antennas and AA in AMCC using the near field method are closest to the process of electrodynamic modeling, and the degree of coincidence of the results corresponds to the accuracy of hardware equipment. A significant advantage of using the AEC is that due to the stable and controlled electromagnetic, mechanical and climatic conditions in the AEC, the measurements of the antennas characteristics and radio equipment are carried out with high accuracy. In addition, the compact size of the AEC allows automation techniques to be used to perform a series of typical measurements, which significantly increases the total productivity of the AMCC. The presence of AEC in the scientific division of the National Research University allows not only to solve the problems posed by scientific and production contracts, but also to implement laboratory workshops in a number of academic disciplines for masters and specialists training.

\section{Acknowledgments}

The study was supported by state assignment of the Ministry of Science and Higher Education of the Russian Federation, project No. FSFF-2020-0015.

\section{References}

[1].Shevgunov, T. (2019, February). A comparative example of cyclostationary description of a nonstationary random process. In Journal of Physics: Conference Series (Vol. 1163, No. 1, p. 012037). IOP Publishing.

[2].Artyushenko, V. M., Volovach, V. I., \& Vasilevich, I. V. (2020, March). Estimation of the Effect of Multiplicative Noise on Signal Detection against the background of Additive Noise. In 2020 Moscow Workshop on Electronic and Networking Technologies (MWENT) (pp. 1-6). IEEE.

[3].Dobychina, E., Malakhov, R., \& Snastin, M. (2014, July). Digital transceiver module for on-board communication system. In 2014 16th International Conference on Transparent Optical Networks (ICTON) (pp. 1-4). IEEE.

[4].Salas-Natera, M. A., Rodriguez-Osorio, R. M., \& de Haro, L. (2012). Procedure for measurement, characterization, and calibration of active antenna arrays. IEEE Transactions on Instrumentation and Measurement, 62(2), 377-391.

[5].Hemming, L. H. (2002). Electromagnetic anechoic chambers: a fundamental design and specification guide. Wiley Interscience.

[6].Dobychina, E. M. (2012, September). Echo-free cellbased antenna measuring and computing complex. In 2012 22nd International Crimean Conference" Microwave \& Telecommunication Technology" (pp. 477-478). IEEE. 
[7].Rodriguez, V., d'Abreu, G., \& Liu, K. (2009, November). Measurements of Power Handling of RF Absorber Materials: Creation of a Medium Power Absorber by Mechanical Means. In 31st Annual Antenna Measurement Techniques Association Annual Symposium (AMTA 2009), Salt Lake City, Utah.

[8].Rodriguez, V. (2018). Validation of a method for predicting anechoic chamber performance: a technique that uses polynomial approximations for RF absorber reflectivity. IEEE Antennas and Propagation Magazine, 60(4), 31-40.

[9].Artyushenko, V. M., \& Volovach, V. I. (2018, January). Synthesis and analysis of discriminators under influence broadband non-Gaussian noise. In IOP Conference Series XI International scientific and technical conference "Applied Mechanics and Dynamics Systems”, J. Phys.: Conf. Ser. (Vol. 944, No. 1, p. 012004).

[10]. Bogaert, I., Olyslager, F., Arien, Y., \& Pissoort, D. (2007, October). Modeling and optimization of advanced multilayered absorbers. In 2007 European Microwave Conference (pp. 214-217). IEEE.

[11]. Hofmann, W., Bornkessel, C., Schwind, A., \& Hein, M. A. (2019, September). Challenges of RF Absorber Characterization: Comparison Between RCS-and NRL-Arch-Methods. In 2019 International Symposium on Electromagnetic Compatibility-EMC EUROPE (pp. 370-375). IEEE.
[12]. Rabelsa, G., Rianto, D., Putra, A., Santosa, C. E., \& Sumantyo, J. T. S. (2019, June). Pyramidal Radar Absorber Based on Coconut Shell Activated Carbon for Anechoic Chamber Application. In 2019 PhotonIcs \& Electromagnetics Research Symposium-Spring (PIERS-Spring) (pp. 1287-1291). IEEE.

[13]. Mishra, N., \& Chaudhary, R. K. (2019). Design and development of an ultrathin triple band microwave absorber using miniaturized metamaterial structure for near-unity absorption characteristics. Progress In Electromagnetics Research, 94, 89-101.

[14]. Gregson, S., McCormick, J., \& Parini, C. (2007). Principles of planar near-field antenna measurements (Vol. 53). IET.

[15]. Maron, M. A. (2018). Diagnostics of Projects. European Research Studies Journal, 21(1), 18-30.

[16]. Russer, P. (2003). Electromagnetics, microwave circuit and antenna design for communications engineering. Artech House.

[17]. Rothwell, E. J., \& Cloud, M. J. (2018). Electromagnetics. CRC press.

[18]. Fang, D. G. (2017). Antenna theory and microstrip antennas. CRC Press. 